\title{
SISTEMAS DE DIREITOS HUMANOS E COMUNIDADES ECONÔMICAS REGIONAIS: UMA DIFERENCIAÇÃO TIPOLÓGICA DOS ATUAIS PROTETORES DE DIREITOS HUMANOS
}

\author{
HUMAN RIGHTS SYSTEMS AND REGIONAL ECONOMIC COMUNITIES: A TYPOLOGICAL \\ DIFFERENCIATION OF CURRENT HUMAN RIGHTS PROTECTORS
}

Marina Feferbaum

Resumo: Diante da presente atuação na proteção de direitos humanos, tanto por parte dos Sistemas Internacionais de Direitos Humanos quanto das Comunidades Econômicas Regionais, neste artigo pretende-se contribuir para uma reflexão sobre as relações entre integração regional e direitos humanos. Por meio da investigação da literatura especializada e do funcionamento prático de alguns exemplos institucionais, foram expostas as diferenças entre os dois arranjos, identificando seus vieses de finalidade e de estrutura. Na primeira parte apresentam-se os sistemas de direitos humanos como centro de análise e tomam-se as comunidades econômicas como parâmetros comparativos. Foram abordados o conceito de sistema, a natureza das organizações internacionais governamentais e as características dimensionais dos sistemas de direitos humanos. Na segunda parte, posicionam-se as comunidades econômicas regionais no centro da investigação, invertendo o foco de análise. $\mathrm{O}$ caso paradigmático de tais comunidades e suas principais características podem ser observados a partir de seu processo e produto. Finalmente, foi realizado um balanço das características dos arranjos e de suas possíveis implicações.

Palavras-chave: Sistemas de direitos humanos. Comunidades econômicas regionais. Direitos humanos. Organizações internacionais. Tipologia.
Abstract: Given the current practice in human rights protection, by both International Systems of Human Rights and Regional Economic Communities, this article aims at contributing to a reflection on the relations between regional integration and human rights. Through an investigation of the literature and of the practical operation of some institutional examples, the differences between the two arrangements were evinced, identifying their purpose's and structures' biases. In the first part human rights systems are presented in the analysis centre and the economic communities are taken as comparative parameters. The concept of system, the nature of governmental international organizations, and dimensional characteristics of human rights systems were addressed. In the second part, the regional economic communities are put at the heart of observation, reversing the focus of the analysis. The paradigmatic case of such communities and their main features can be observed from the perspective of both its process and product. Finally, it was balanced the characteristics of each arrangement and their possible implications.

Keywords: Human rights systems. Regional economic communities. Human rights. International organizations. Typology.

\footnotetext{
* Mestre e doutoranda em Direito pela Pontifícia Universidade Católica de São Paulo; Coordenadora da área de Metodologia de Ensino da FGV DIREITO SP; Rua Rocha, 233, Bela Vista, 01330-000, São Paulo, São Paulo, Brasil; marina.feferbaum@fgv. br
} 


\section{Introdução}

A expansão da tutela dos direitos humanos com a qual se lida atualmente vem acontecendo desde 1948, quando foram inaugurados, do ponto de vista institucional, o discurso e o paradigma corrente da matéria. Desde então, ocorreu a adoção das cartas universal e regionais de direitos humanos, seguida da instauração dos grandes sistemas de promoção e proteção de direitos humanos e da criação de tribunais especializados. Porém, diferentemente do que ocorreu nas primeiras seis décadas do século XXI, o crescimento atual dos aparatos de proteção dos direitos humanos tem contado com arranjos internacionais diversos, e a atual defesa internacional dos direitos humanos já não se restringe aos grandes sistemas de proteção especializada. Diferentes iniciativas têm colocado as Comunidades Econômicas Regionais (CERs), concebidas originalmente para conduzir a integração de mercados, no mapa de proteção desses direitos.

De fato, observa-se uma expansão de pautas de direitos humanos em várias Comunidades Econômicas Regionais (CERs), como vem ocorrendo no Mercosul, na Associação de Nações do Sudeste Asiático (Asean), na Comunidade Econômica dos Estados da África Ocidental (CEDEAO ou ECOWAS), entre outros. Com crescente frequência, os tratados de constituição dessas comunidades (ou cartas comunitárias) fazem referência preambular ou dispositiva ao respeito a direitos humanos, atribuindo-lhes, por vezes, a qualidade de valor fundamental ou de diretriz do bloco. ${ }^{1}$ Observa-se também o surgimento de órgãos, de grupos de trabalhos, entre outros, especializados em direitos humanos e temas relacionados. ${ }^{2}$ Em alguns episódios, os tribunais comunitários passaram, inclusive, a exercer jurisdição sobre casos envolvendo a matéria. ${ }^{3}$

\footnotetext{
1 Alguns exemplos são:

- Excerto do preâmbulo da Carta da Asean (ASSOCIAÇÃO DE NAÇÕES DO SUDESTE ASIÁTICO, 2007): "ADERÊNCIA aos princípios da democracia, Estado de Direito e boa governança, respeito e proteção dos direitos humanos e liberdades fundamentais." (Trad. livre de: "ADHERING to the principles of democracy, the rule of law and good governance, respect for and protection of human rights and fundamental freedoms.")
}

- Excerto do preâmbulo da Carta da CEDEAO (COMUNIDADE ECONÔMICA DOS ESTADOS DA AMÉRICA OCIDENTAL, 2010): "TENDO EM MENTE a Carta Africana dos Direitos dos Homens e dos Povos e a Declaração de Princípios da Comunidade Econômica dos Estados da África Ocidental adotada na 14 ${ }^{a}$ Sessão Ordinária da Conferência dos Chefes de Estado e de Governo em 6 de julho de 1991 em Abuja." (Trad. livre de: "BEARING IN MIND the African Charter on Human and People's Rights and the Declaration of Political Principles of the Economic Community of West African States adopted in Abuja by the Fourteenth Ordinary Session of the Authority of Heads of State and Government on 6 July, 1991.");

- Excerto do preâmbulo da Carta da SADC (SOUTHERN AFRICA DEVELOPMENT COMMUNITY, 1992): "CONSCIENTE da necessidade de envolver os povos da Região centralmente no processo de desenvolvimento e integração, particularmente pela garantia dos direitos democráticos, observância dos direitos humanos e do estado de direito." (Trad. livre de: "MINDFUL of the need to involve the peoples of the Region centrally in the process of development and integration, particularly through the guarantee of democratic rights, observance of human rights and the rule of law.");

Carta da SADC, Artigo 4 (SOUTHERN AFRICA DEVELOPMENT COMMUNITY, 1992): "PRINCÍPIOS. SADC e seus Estados Membros deverão atuar conforme os seguintes princípios: [...] c) direitos humanos, democracia e o estado de direito" (Trad. Livre de: "SADC and its Members States shall act in accordance with the following principles: [...] c) human rights, democracy and the rule of law;").

2 Alguns exemplos são encontrados:

- No Mercosul, a Reunião de Altas Autoridades de Direitos Humanos (RAADDHH), criada pela Decisão n. 40/04 do Conselho do Mercado Comum (CMC) (MERCOSUL/CMC/DEC. n. 40/04), e o Instituto de Políticas Públicas em Direitos Humanos (IPPDH), criado pela Decisão n. 14/09 do CMC (MERCOSUL/CMC/DEC. n. 14/09);

- $\mathrm{Na}$ Asean, a Comissão Intergovernamental da Asean sobre Direitos Humanos (AICHR);

- Na CEDEAO, o Centro do Desenvolvimento do Gênero da CEDEAO (CDGC).

3 CEDEAO, EAC e SADC (até 2010, quando foi suspensa a jurisdição sobre casos de direitos humanos). 
A existência de questões de direitos humanos em comunidades econômicas não é exatamente uma novidade da atualidade, já que casos com algum tipo de relação a esses direitos remontam à criação da CECA, em 1952, ${ }^{4}$ multiplicando-se na Comunidade Econômica Europeia (CEE), estabelecida em 1957 com a Comunidade Europeia de Energia Atômica (Euratom) pelo Tratado de Roma. ${ }^{5}$ Mas foi apenas recentemente que o tema ganhou saliência em algumas comunidades do ponto de vista institucional, como nos exemplos evidentes das tratativas para a adesão da União Europeia ao Sistema Europeu de Direitos Humanos e da ampliação da competência do Tribunal de Justiça da Comunidade Econômica dos Estados da África Ocidental (CEDEAO ou ECOWAS) para lidar com a matéria.

O papel desses novos atores ainda não é claro. Seriam eles versões menores dos tradicionais sistemas regionais? Seriam complementares ou concorrentes desses últimos? Haveria algum tipo de interferência proveniente do propósito institucional, ou seja, haveria um viés econômico no tratamento dos direitos humanos? Partindo dessa inclusão das Comunidades Econômicas Regionais no ambiente político de discussões de direitos humanos, o objetivo com este artigo é identificar semelhanças e diferenças institucionais entre os grandes sistemas de direitos humanos e essas Comunidades, como um primeiro passo na direção da resposta a essas perguntas. Tais aspectos são determinantes para a compreensão posterior dos respectivos papéis na proteção internacional dos direitos humanos e para tornar mais claras as limitações de cada tipo de arranjo nessa atuação.

Este trabalho passa por elementos teóricos e práticos (do próprio funcionamento de exemplos institucionais) para compor um quadro de elementos distintivos entre sistemas de direitos humanos e Comunidades Econômicas Regionais. Na primeira parte, são discutidos alguns elementos comuns dos sistemas de direitos humanos, colocando-os como centro da análise. Foram abordados o conceito de sistema, a natureza das organizações internacionais governamentais e as características dimensionais dos sistemas de direitos humanos. Na segunda parte, a diferenciação tipológica é complementada invertendo o foco de análise: as comunidades econômicas passam à posição central de objeto de observação, enquanto os sistemas de direitos humanos se tornam parâmetro comparativo. O caso paradigmático das Comunidades Econômicas é apresentado, seguido de uma análise pela perspectiva do processo e do produto das comunidades. Nas considerações finais, é realizado um balanço dos resultados desse exercício tipológico e de suas possíveis implicações.

\footnotetext{
4 Cf. Case n. 1/58. Friedrich Stork \& Cie v. High Authority of the European Coal and Steel Community (EUROPEAN COMMUNITIES, 1959).

5 O Tratado de Roma, na realidade, são dois tratados distintos: o Tratado de Constituição da Comunidade Econômica Europeia (EUROPEAN ECONOMIC COMMUNITY, [s.d.]), disponível em versão oficial nos idiomas francês, holandês e italiano, e o Tratado de Constituição da Comunidade Europeia de Energia Atômica (UNIÃO EUROPEIA, 2010), disponível em versão oficial nos idiomas alemão, francês, holandês, inglês e italiano.
} 


\section{Sistemas de direitos humanos: alguns elementos comuns}

A transformação dos sistemas de direitos humanos em modelos institucionais de referência para o direito internacional pode estar relacionada, em grande parte, ao estabelecimento de alguns dos seus grandes exemplares, concomitantemente ao surgimento do discurso contemporâneo de direitos humanos. Por essa razão, quando se pensa na estrutura institucional de defesa de direitos humanos, pensa-se, provavelmente, nos grandes sistemas, seja ocupando seu centro (isto é, como o principal motor de movimentação da estrutura), seja ocupando sua totalidade (isto é, como único motor, sem estruturas secundárias ou periféricas).

Claro que as diferenças existentes entre os chamados sistemas de direitos humanos não são poucas, mas seus aspectos comuns são suficientes para se conceber um modelo genérico daquilo que se convencionou chamar de sistema. Para os elementos comuns desses sistemas de direitos humanos ficarem visíveis, são abordados três aspectos. Primeiro, o conceito de sistema quando utilizado no emprego como sistema de direitos humanos. No segundo, é explorada a natureza de tais organizações internacionais (OIGs). Finalmente, são traçadas as suas características dimensionais, que completam o quadro de identificação do que são os sistemas de direitos humanos.

\subsection{Conceitos de sistema}

Neste ponto, é essencial retomar à função de se investigar o conceito de sistema para se entenderem diferenças institucionais. A pergunta que importa é basicamente: há, entre os recentes arranjos (como das Comunidades Econômicas Regionais), novos tipos de sistemas de direitos humanos, além do global e dos cinco regionais existentes e conhecidos como tais? A pergunta já foi analisada por Viljoen (2007, p. 10), a partir de Freeman, e serve aqui como reflexão acerca do recorte jurídico da matéria. ${ }^{6}$ As balizas dessa reflexão foram traçadas em três acepções: sistema como um

\footnotetext{
6 Na seguinte passagem, Viljoen (2007, p. 10), com referência a Freeman (2002, p. 53), afirma a inexistência de sistemas sub-regionais de direitos humanos: "Normalmente, arranjos sub-regionais são destinados primariamente à integração econômica, e não à promoção e proteção de direitos humanos. Haja vista que eles funcionam em um nível cujo liame interestatal pode ser considerado relativamente forte, o potencial desses arranjos para direitos humanos é evidente. Não obstante nenhum sistema sub-regional de direitos humanos exista na África, há um número de conjuntos intergovernamentais que incluem preocupação com direitos humanos em seus mandatos." Trad. livre de: "Usually, subregional arrangements are primarily directed at economic integration, and not at the promotion and protection of human rights. Given that they function at a level which inter-country bonds may be assumed to be relatively strong, the potential of these arrangements for human rights is evident. Although no subregional human rights system exists in Africa, there are a number of subregional intergovernmental groupings that include a concern for human rights within their mandates." Murungi e Gallinetti (2010, p. 124) esclarecem a afirmação de Viljoen: "Um sistema de direitos humanos consiste em um conjunto de normas e instituições aceitas pelos estados como vinculantes (FREEMAN, 2002, p. 53). Analisados perante tal definição, os esforços das comunidades econômicas regionais (CERs) atinentes aos direitos humanos não se reputam sistemas de direitos humanos independentes. Isso porque, apesar das frequentes referências aos direitos humanos, eles não possuem instituições estabelecidas especificamente para lidar com direitos humanos. Essa é a base do argumento de que não há na África sistemas sub-regionais de direitos humanos, senão apenas arranjos sub-regionais interestatais com preocupação com os direitos humanos em seus mandatos (VILJOEN, 2007, p. 10)". Trad. livre de "A human rights system consists of a set of norms and institutions accepted by states as binding (FREEMAN, 2002, p. 53). Assessed against such a system, the efforts of RECs with respect to human rights fall short of constituting independent human rights systems. This is because despite making extensive references to human rights, they lack corresponding institutions established specifically to deal with human rights. This is the basis of the argument that there are no sub-regional human rights systems existing in Africa but that they are simply sub-regional intergovernmental groupings with human rights as a concern within their mandate." (VILJOEN, 2007, p. 10)."
} 
objetivo da organização (único ou não), sistema como maquinário jurídico-administrativo e sistema como organograma interorganizacional.

Sem dúvida, o primeiro é o uso mais comum da expressão e se liga à ideia de conjunto de elementos (instrumentos, mecanismos, discursos, etc.) relacionados à defesa de direitos humanos em determinada organização. Nesse sentido, o termo reflete a própria organização internacional governamental: onde se diz "sistema interamericano", por exemplo, deve-se ler "arcabouço de direitos humanos da OEA". Em outros temos, aquilo que confere a unidade sistêmica é a própria organização.

Dentro do segundo sentido, relacionado ao maquinário jurídico administrativo, importam as qualificações geral e especial. Se há um sistema global geral e sistemas globais especiais, é porque não há um sistema global único, divergindo da acepção anterior. Nesse caso, a expressão exprime um conjunto específico de instrumentos, órgãos e mecanismos, que juntos formam um todo coerente. Por exemplo, o sistema especial contra discriminação da mulher, instituído pela Convenção sobre a Eliminação de Todas as Formas de Discriminação contra a Mulher, possui disposições jurídicas específicas, mecanismo de relatório e de petições individuais, ${ }^{7}$ e um comitê próprio para apreciação das petições (UNITED NATIONS, 1980). Em suma, a unidade sistêmica emerge do tratado (incluindo eventuais protocolos), que cria toda a estrutura necessária para o seu funcionamento. A unidade, nessa perspectiva, ostenta autonomia operacional e existencial, exprimindo um conceito mais estrito de sistema.

terceiro uso da expressão diz respeito ao conjunto formado de organizações, órgãos autônomos e entidades diversas que orbitam a organização principal, também incluída. Equivale à imagem do sistema formado por um planeta e seus satélites ou de um sistema planetário. É o caso do sistema da Organização das Nações Unidas (ONU), no qual dezenas de agências, fundos, programas e organizações correlatas formam com a primeira uma identidade comum. É a unidade sistêmica decorrente de uma vinculação institucional umbilical.

Essa última acepção, de fato, destoa das demais. Ela é específica da expressão Sistema das Nações Unidas ${ }^{8}$ (que exprime o "modelo planetário" da organização), a qual não possui qualquer especificidade relacionada aos direitos humanos, apresentando tão somente um significado relacionado à estrutura da organização. Ela não se confunde com a ideia de sistema de direitos humanos da $O N U$ atinente à matéria. Essa acepção diversa do termo sistema dá visibilidade às atividades da organização principal que são desenvolvidas fora dela. A Organização das Nações Unidas para a Educação, a Ciência e a Cultura (Unesco), por exemplo, é uma organização autônoma da ONU, cuja atividade segue os propósitos desta quanto a questões educacionais, científicas e culturais, como "[...] a forma-

\footnotetext{
7 Instituído pelo Protocolo Facultativo à Convenção. Cf. Piovesan (2009, p. 203).

8 "O Sistema da ONU, também denominado informalmente como 'Família ONU', é composta pela própria ONU e muitos programas afiliados, fundos e agências especializados, todos com adesão, direção e orçamento próprios. Os programas e fundos são financiados por contribuições voluntárias ao invés de compulsórias. As agências especializadas são organizações internacionais independentes, financiadas por contribuições tanto voluntárias como compulsórias". Trad. livre de: "The UN system, also known unofficially as the 'UN family', is made up of the UN itself and many affiliated programmes, funds, and specialized agencies, all with their own membership, leadership, and budget. The programmes and funds are financed through voluntary rather than assessed contributions. The Specialized Agencies are independent international organizations funded by both voluntary and assessed contributions." (UNITED NATIONS, 2015).
} 
ção de professores, o melhoramento da educação em nível mundial e a proteção de locais históricos e culturais importantes ao redor do mundo." (UNITED NATIONS, 2015). ${ }^{9}$ É, portanto, um prolongamento da ONU, mas com personalidade jurídica própria. Com a Unesco, há mais de uma dezena de "organizações satélite" da ONU, oficialmente denominadas agências especializadas, entre as quais várias com propósito diretamente relacionado à promoção de direitos humanos, a saber: a própria Unesco; a Organização Internacional do Trabalho (OIT), que promove o "[...] trabalho decente a todos pelo estabelecimento de normas internacionais sobre a liberdade de associação, a negociação coletiva, a abolição do trabalho forçado e a igualdade de oportunidades e de tratamento." (UNITED NATIONS, 2015); ${ }^{10}$ a Organização Mundial da Saúde (OMS), responsável por campanhas mundiais de vacinação, gestão de emergências de saúde pública, entre outras (UNITED NATIONS, 2015); a Organização das Nações Unidas para a Alimentação e a Agricultura (FAO), que lidera a "luta contra a fome no mundo" (UNITED NATIONS, 2015); o Fundo Internacional de Desenvolvimento Agrícola (FIDA ou IFDA), que "[...] trabalha exclusivamente com as populações rurais pobres dos países em desenvolvimento para ajudá-las a sair da pobreza, a garantir sua segurança alimentar e nutricional, a aumentar sua produtividade e renda e a melhorar sua qualidade de vida" (UNITED NATIONS, 2015);11 e a Organização das Nações Unidas para o Desenvolvimento Industrial (ONUDI ou UNIDO), "[...] encarregada de promover o desenvolvimento industrial para a redução da pobreza [...]" (UNITED NATIONS, 2015). ${ }^{12}$

A "estrutura planetária" do Sistema das Nações Unidas pode dar a falsa impressão de que seus "satélites" são apenas especializações temáticas com autonomia administrativa e financeira, concebidas sob forma organizacional, o que seria uma generalização não correspondente às respectivas histórias institucionais. De fato, somente parcela das agências foi concebida pela ONU. Algumas delas são mais antigas (caso da OIT, de 1919, e da FAO, de 1943), o que coloca a promoção de direitos humanos por meio de organizações internacionais governamentais como atividade surgida no entreguerras. Haveria, então, alguma diferença substancial entre os direitos humanos desse período e o do pós-guerra?

A resposta é simples: as declarações de 1948 introduziram uma nova perspectiva para a matéria, visto que atribuíram a titularidade dos direitos (humanos) ao indivíduo, independentemente do suporte de uma ordem jurídica nacional. Sua adoção como soft law em nada afeta a referência histórica, já que a mudança ocorre justamente no discurso axiológico dos direitos humanos, e não na sua ordem positiva. O primeiro sistema surge anos mais tarde, em 1953, com a entrada em vigor da Convenção Europeia de Direitos Humanos (1950/1953) (COUNCIL OF EUROPE, 1950), que inaugurou o Sistema Europeu. Surgiram, em seguida, o Sistema Global (1966-1976) (UNITED NA-

\footnotetext{
9 Trad. livre de: "The United Nations Educational, Scientific and Cultural Organization focuses on everything from teacher training to helping improve education worldwide to protecting important historical and cultural sites around the world." A versão em francês do site também foi consultada para elaboração da tradução.

10 Trad. livre de: "[...] promotes international labor rights by formulating international standards on the freedom to associate, collective bargaining, the abolition of forced labor, and equality of opportunity and treatment."

11 Trad. livre de: "[...] has focused exclusively on rural poverty reduction, working with poor rural populations in developing countries to eliminate poverty, hunger and malnutrition; raise their productivity and incomes; and improve the quality of their lives."

12 Trad. livre de: "[...] promotes industrial development for poverty reduction, inclusive globalization and environmental sustainability."
} 
TIONS, 1967a, 1967b), o Sistema Interamericano (1969-1978) (ORGANIZATION OF AMERICAN STATED, 1969), o Sistema Africano (1981-1986) (ORGANIZATION OF AFRICAN UNITY, 1982) e o Sistema Árabe (2008) (LEAGUE OF ARAB STATES, 2005). A referência, em todos esses casos, foi a entrada em vigor das respectivas cartas de direitos humanos, o que remete, a princípio, à segunda acepção de sistema.

$\mathrm{Na}$ realidade, observa-se que a existência de um tratado fundador é um critério implícito de identificação de um sistema como tal, mas não basta como definição. Em outras palavras, um sistema de direitos humanos assim se reputa se possui um tratado de direitos humanos que estabeleça direitos individuais, mecanismos de controle e competência jurisdicional, os quais, no entanto, não delimitam o próprio sistema. Daí a primeira acepção de sistema, que se confunde com a organização que alberga os mecanismos. De todo modo, nota-se uma dependência conceitual da primeira com a segunda acepção, já que a inexistência de contornos evidentes entre OIG e sistemas de direitos humanos da primeira acepção não exclui do conceito o requisito de uma estrutura nuclear formada pelo trinômio direito-mecanismo-órgão, instituído por um tratado de direitos humanos.

\subsection{Organizações internacionais governamentais e sua natureza}

Um segundo aspecto dos sistemas de direitos humanos diz respeito ao tipo (ou natureza) de sua OIG. Os grandes sistemas operam sobre organizações conduzidas por agendas de política internacional, ou seja, voltadas às relações internacionais em si, o que, por sua vez, é de difícil definição ante a inexistência de contornos evidentes desse significado quanto à forma e à área de atuação. Assim, os sistemas de direitos humanos podem compreender questões de paz, segurança, democracia, direitos humanos, meio ambiente, educação, trabalho, acesso à água, energia, transporte, além de inúmeras outras.

De toda forma, os grandes sistemas de direitos humanos funcionam em OIGs que são organizações políticas. Isso permite já uma distinção clara das CERs, cuja atividade é de integração econômica. Essa distinção é relativamente frequente na literatura sobre regionalismo (FAWCETT, 2003, p. 17 e ss.) como forma de agrupar em categorias gerais os diferentes tipos de arranjos da década de 1960, definidas conforme o tipo de agenda. Com isso, passou-se a distinguir as agendas políticas das agendas de segurança e econômica.

Essa distinção é menos evidente na atualidade, em parte por conta da intersecção de agendas em muitas OIGs. É o caso das discussões de projetos de desenvolvimento econômico que ocorrem, por exemplo, na OEA. Ou mesmo no caso das CERs que possuem jurisdição sobre violações de direitos humanos, as quais compreendem ambas a agenda econômica da integração e a agenda política da defesa de direitos humanos.

Do ponto de vista teórico, é tentador relacionar acordos de integração econômica ou de defesa militar como empreitadas políticas, esvaziando quase totalmente a diferença entre as categorias. Essa discussão será retomada ao tratar das CERs, bastando, por ora, enquadrar as OIGs dos grandes sistemas sob o grupo das organizações políticas. 
Em organizações do tipo, há o propósito amplo de comunicação entre os membros. Por esse ângulo, observa-se a existência de três tipos de diálogo: diálogo de conciliação, diálogo de deliberação e diálogo de fomento. $O$ primeiro diz respeito às boas relações entre os membros, o que remete à promoção da paz. O segundo refere-se aos interesses comuns que dependem de esforços conjuntos para se alcançarem objetivos estabelecidos por consenso. $O$ terceiro liga-se ao desenvolvimento humano, no qual valores atinentes às liberdades instrumentais ${ }^{13}$ são promovidos.

Seja qual for o tipo de diálogo que promove sua natureza, os objetivos dessa interlocução são variados, desde o fomento de cooperação até a negociação de paz em conflitos. Por essa ótica, uma organização política internacional governamental (OPIG) funciona como um grande fórum político, no qual os Estados debatem as mais variadas matérias. A Assembleia Geral da ONU é particularmente ilustrativa dessa ideia.

É difícil ir além dessa abordagem geral, na medida em que as OPIGs dos grandes sistemas são bastante diversificadas, principalmente em relação às matérias das respectivas agendas. Essa diversidade também se reflete nos direitos humanos, cada qual com particularidades de diplomas, mecanismos e funcionamento. Desse grupo - composto por ONU, OEA, Conselho da Europa, União Africana e, com algumas ressalvas, a Liga Árabe - destaca-se o caso da União Africana (UA) por compreender em sua agenda atual um projeto de integração econômica, que não existia quando da instalação do Sistema Regional Africano.

De maneira breve, a UA é sucessora da Organização da Unidade Africana (OUA), estabelecida na década de 1960. Na década de 1990, foi criada a Comunidade Econômica Africana (AEC, segundo a denominação em inglês), um projeto de integração econômica de proporções continentais. Formalmente, a AEC não se confundia com a OUA, embora compartilhasse toda a infraestrutura material desta última. Por essa razão, a OUA passou a ser referida como OUA/AEC, inclusive pelo Tratado da UA. Assim, a análise do enquadramento da AEC em relação à agenda, isto é, como OPIG ou CER, para fins de enquadrar a UA, envolve outras considerações, que são tratadas nos próximos itens.

\subsection{Características dimensionais}

Um terceiro aspecto que auxilia a identificação de sistemas de direitos humanos, especialmente em relação às CERs, diz respeito à abrangência do conjunto de membros, o que envolve, entre outros elementos, território, população e quantidade de países. São grandezas que podem expressar tamanho em suas várias acepções, entre as quais aquelas que decorrem da distinção regional/global, utilizada para distinguir o sistema da ONU dos demais, e que pode servir como parâmetro para diferenciar regiões dentro de regiões, como é o caso das comunidades econômicas.

\footnotetext{
13 "Cinco tipos distintos de liberdade vistos de uma perspectiva 'instrumental' são investigados particularmente nos estudos a seguir. São eles: (1) liberdades políticas, (2) facilidades econômicas, (3) oportunidades sociais, (4) garantias de transparência e (5) segurança protetora. Cada um desses tipos distintos de direitos e oportunidades ajuda a promover a capacidade geral de uma pessoa. [...] Na visão do 'desenvolvimento como liberdade', as liberdades instrumentais ligam-se umas às outras e contribuem com o aumento da liberdade humana em geral." (SEN, 2000, p. 25).
} 
A abrangência do conjunto de membros de determinada OIG denota, antes de tudo, o tamanho. Utilizar o volume de membros como parâmetro da tipologia de OIG pode ser particularmente problemático, já que há muitas variáveis envolvidas no conceito (como o número de estados, o tamanho da população e a extensão da área), dificultando o estabelecimento de referências numéricas adequadas. A China sozinha, por exemplo, é mais populosa que qualquer um dos sistemas regionais de direitos humanos. Por isso, o tamanho é uma característica relativa, cujo significado deve ser analisado caso a caso. É, também, uma característica eventual, que não determina a natureza da OIG. Sua relevância depende da escala, na medida em que quanto maiores forem as grandezas numéricas do conjunto de membros de uma OIG, maior será a heterogeneidade envolvida. Em outras palavras, embora não sejam determinantes e devam ser consideradas com cautela, as características dimensionais de uma OIG têm significado, ainda que limitado.

Tomando como ponto de partida uma reflexão sobre tamanho e divisão da política internacional entre os continentes, a qual retrata a circunscrição de três dos grandes sistemas (Sistema Americano, Sistema Europeu e Sistema Africano de Direitos Humanos), observa-se certo equilíbrio entre África, Américas e Europa na quantidade de países e população (Tabela 1).

Tabela 1 - Distribuição da população mundial em 2013 por continente

\begin{tabular}{lll}
\hline CONTINENTE & PAÍSES $^{*}$ & POPULAÇÃO* \\
\hline África & 58 & $1.110,6$ \\
Américas & 53 & 972,0 \\
Ásia & 50 & $4.298,7$ \\
Europa & 50 & 742,5 \\
Oceania & 25 & 38,3 \\
\hline TOTAL & 236 & $7.162,1$ \\
\hline
\end{tabular}

Fonte: United Nations (2014).

Notas: * Independentes ou não. Macau e Hong Kong foram considerados separadamente da China. ${ }^{* *}$ Em milhões de habitantes.

Ainda que com um pouco mais de variação, esse equilíbrio permanece em relação aos respectivos sistemas regionais (Tabela 2).

Tabela 2 - População por organização

\begin{tabular}{lll}
\hline OIG & MEMBROS & POPULAÇÃO* \\
\hline Conselho da Europa & 47 & 800 \\
Liga Árabe & 21 & 370 \\
OEA & 35 & 970 \\
União Africana & 54 & 1.000
\end{tabular}

Fonte: United Nations (2014) e Council of Europe (2013).

Nota: * Em milhões de habitantes.

Comparativamente a algumas CERs, a União Europeia possui, aproximadamente, 500 milhões de habitantes em 28 países, e o Mercosul, aproximadamente, 300 milhões de habitantes em seis países. Um mapeamento das CERs mostra que são poucas as comunidades com mais de 20 países; uma parcela significativa tem menos de 10. Com a notável exceção da AEC, todas as CERs são de escala sub-regional, entendidas como de circunscrição subcontinental, que se contrapõe aos 
três principais sistemas regionais de direitos humanos. A esse propósito, nota-se que as OPIGs dos sistemas de direitos humanos são, geralmente, maiores que as CERs.

Como já mencionado, o critério tamanho é relativo, a exemplo da comparação entre UE (uma CER com 500 milhões) e a Liga Árabe (uma OPIG, com 370 milhões). Contudo, há razões para diferenças em decorrência da agenda. Embora uma OPIG possa ser menor que uma CER, seu tamanho limita-se apenas pelo seu critério de adesão, por exemplo, os Estados do continente europeu, no caso da União Europeia. Por essa premissa, uma OPIG poderia comportar todos os Estados do globo sem prejudicar sua capacidade operacional. A CER, ao contrário, tem limites impostos pelo próprio projeto, podendo não comportar a adesão de um determinado Estado em razão do risco a que sua economia pode expor o mercado comunitário.

Admitindo esse raciocínio, a Comunidade Econômica Africana representa um projeto inviável, já que o projeto de integração regional foi concebido como uma comunidade aberta à adesão de todos os países do continente. É uma missão de difícil consecução, embora sua estratégia de promover uma integração "mosaico", a partir de comunidades menores, consiga resultados em escala sub-regional:

A magnitude da tarefa para alcançar crescimento econômico e desenvolvimento integrados na África não deve inspirar um impasse, devendo-se destacar o fato que esforços devem ser direcionados ao nível sub-regional como um ponto de partida lógico e necessário, alinhado tanto com o Ato Constitutivo da UA quanto com o Tratado da AEC. Essa dimensão mais modesta da unificação confere um cenário muito mais plausível para uma estória de sucesso. Proponentes da integração e unidade africana veem o redesenho das fronteiras loteadas na Conferência de Berlim como um importante passo para um novo começo. Em vez de reimaginar Berlin e reorganizar o mapa africano, deve-se aceitar que algumas comunidades econômicas regionais (RECs) dominantes serão, eventualmente, os elementos fundantes de uma nova soberania pan-africana. Mais do que simples elementos, elas devem, no curto prazo, ser o foco e o ponto final da evolução daquilo que é factível e possível, deixando a unidade em escala pan-africana como uma perspectiva muito mais remota. (VILJOEN, 2012, p. 481-482). ${ }^{14}$

O projeto da AEC desafia as demais propostas de integração pela razão já apresentada. De fato, as demais iniciativas existentes, incluindo a UE, partem ou partiram de agrupamentos menores, seguindo um processo paulatino de integração para atingir determinada profundidade, conforme cada pretensão. A adesão de novos membros sempre seguiu com cautelas, envolvendo um longo

\footnotetext{
14 Trad. livre de: "The magnitude of the task to achieve integrated economic growth and development in Africa should not inspire an impasse, but should rather highlight the fact that efforts must be directed at the subregional level as a logical and necessary starting point, in line with both the AU Constitutive Act and AEC Treaty. This more modest dimension of unification provides a much more likely setting for a success story. Some proponents of African integration and unity view the re-drafting of the Berlin-brokered boundaries as an important step towards a new beginning. Rather than re-imagining Berlin, though, and playing a reconfiguration numbers game, it should be accepted that a few dominant regional economic communities (RECS) will eventually be the constitutive elements of a new pan-African statehood. More than mere building blocks, they should in the short term form the focus and the end point of the evolution of what is feasible and possible, leaving unity on a pan-African scale as a much more remote prospect." A reflexão de Viljoen na passagem é um pouco diferente do sentido em que foi citado. $O$ autor reflete sobre duas posições opostas: uma pela unificação do continente; outra, por uma nova divisão do continente, ante à inviabilidade do primeiro. $O$ autor segue o caminho intermediário da integração sub-regional, que é justamente a premissa da integração continental, que reputa a um objetivo bastante distante. A conclusão, no entanto, serve ao propósito da citação.
} 
processo de homologação. No caso da AEC, logo após a adoção de seu tratado, praticamente todos os estados do continente apresentaram adesão antes que qualquer passo fosse dado na sua consecução. Passadas mais de duas décadas, não existe comunidade, se não suas estruturas organizacionais, muitas já existentes quando da sua criação.

Deve-se observar que a relação OUA/AEC nunca foi muito clara. Ambas as instituições surgiram com uma ideologia pan-africanista. A criação da OUA em 1963 teve como objetivo descolonizar o continente africano e combater o apartheid (FEFERBAUM, 2012, p. 72). Com praticamente todos os objetivos alcançados, concebeu-se, então, a AEC em 1991 para o próximo passo, isto é, a unificação do continente. O modelo, certamente, inspirou-se na então Comunidade Econômica Europeia, tanto no propósito quanto no nome. Posteriormente, com o fim de reestruturar a antiga OUA (e AEC), foi criada a União Africana, novamente inspirada no novo nome do projeto europeu.

Ainda atualmente, a relação UA/AEC não possui contornos bem definidos, embora, na prática, toda deliberação aconteça em conjunto. Essa indefinição, a princípio, dificulta o enquadramento como OPIG ou como CER, embora, ante a inexistência de uma comunidade continental concreta, possa-se reputar à comunidade um objetivo programático, considerando-se o arranjo como uma agenda política.

\section{Comunidades econômicas regionais}

Conforme já mencionado, falar em Comunidade Econômica Regional (CER) é referir-se a uma agenda econômica. Com a incorporação de pautas políticas nessas comunidades e de discussões de questões econômicas em OPIGs, a diferença entre agendas tem-se tornado mais estreita, de mais difícil distinção, como no caso OUA/AEC, atualmente UA. O objetivo nesta seção é, então, investigar a integração regional a fundo para buscar um melhor entendimento dos limites dessa categoria.

\subsection{0 caso paradigmático: União Europeia}

A integração regional é um tema que surge com o estudo da integração europeia, que remonta à criação da Comunidade Econômica do Carvão e do Aço (CECA) em 1952. Desde então, as teorias de integração foram desenvolvidas sobretudo para explicar o caso europeu, ${ }^{15}$ o mais adiantado entre os existentes. Por essa razão, o estudo da integração regional por uma perspectiva teórica é, antes de tudo, um estudo da União Europeia. Por um lado, isso fornece uma projeção de um estágio avançado de integração e do respectivo caminho percorrido; por outro, pode se diferenciar da realidade e do caminho dos demais projetos de integração regional em curso.

15 "Theories of integration have mainly been developed to explain European integration." (LAURSEN, 2008, p. 3). 
A União Europeia não pode ser compreendida a partir de um conceito tradicional, pois ela é um "animal novo", ${ }^{16}$ um tipo de entidade jurídico-política surgida na segunda metade do século $\mathrm{XX}$, que é, em si, representante de uma categoria de difícil compreensão. Consequentemente, é, da mesma forma, difícil evitar comparações com referências mais familiares na tentativa de descrevê-la. Sobre isso, McCormick e Olsen (2014) observam:

A maneira pela qual abordamos a UE ainda depende em grande parte de como pensamos acerca do papel do Estado. Consequentemente, há pelo menos cinco maneiras de conceituar a UE - como uma organização internacional, uma associação de integração regional, um sistema político à sua própria maneira, uma entidade única ou algo que combine todas as anteriores. ${ }^{17}$

Entretanto, qualquer que seja a referência adotada, o resultado final decorrerá de um contato parcial com o objeto, que pode ser pouco representativo do seu conjunto total, tal qual já alertava a antiga parábola indiana sobre o desconhecido, reproduzida por Puchala (1972, p. 267) no artigo Of Blind Men, Elephants and International Integration:

Vários cegos se aproximaram dum elefante e cada um tocou o animal no esforço de descobrir sua aparência. Cada cego, contudo, tocou uma parte diferente do grande animal, e cada um concluiu que o elefante tinha a aparência da parte tocada. Logo, o homem que sentiu a tromba conclui que o elefante teria de ser alto e esbelto, enquanto o que tocou a orelha conclui que seria alongado e plano. Os outros, é claro, chegaram a diferentes conclusões. O resultado foi que nenhum homem chegou a uma descrição precisa do elefante. Entretanto, cada homem obteve de sua própria experiência evidência suficiente para discordar de seus companheiros e para sustentar um vigoroso debate sobre a natureza da besta. ${ }^{18}$

A União Europeia é este animal desconhecido que ninguém consegue ver diretamente, o que faz com que cada um tente descrevê-lo a partir das referências individuais. Há, por conseguinte, muitas abordagens possíveis, cada qual com algum tipo de limitação. Talvez não seja possível formar, de maneira concisa, uma imagem acurada da sua natureza; contudo, é possível concebê-la a partir daquilo que ela não é, já que as limitações das referências familiares representam desvios das respectivas classificações.

\footnotetext{
16 A expressão "novo animal" foi utilizada por O’Donnell para introduzir o conceito de democracia delegativa, "[...] um subtipo das democracias existentes, que ainda há de ser teorizada." (Trad. livre de: "[...] a subtype of existing democracies, which has yet to be theorized." (O’DONNELL, 1993). Na mesma linha, McCormick e Olsen (2014) descrevem a UE como "[...] um animal de uma linhagem diferente: é um arranjo político que desafia uma definição ou categorização simples e não se encaixa em ideias ortodoxas de política e governo." (Trad. livre de: "[...] but the European Union is an animal of a different stripe: it is a unique political arrangement that defies easy definition or categorization and does not fit orthodox ideas about politics and government."

17 Trad. livre de: "How we approach the EU still depends in large part on how we think about the role of the state. Consequently, there are at least five ways to conceptualize the $E U$ - as an international organization, a regional integration association, a political system in its own right, a unique entity, or something that exhibits and combines all four of these."

18 Trad. livre de: "Several blind men approached an elephant and each touched the animal in an effort to discover what the beast looked like. Each blind man, however, touched a different part of the large animal, and each concluded that the elephant had the appearance of the part he had touched. Hence, the blind man who felt the animal's trunk concluded that an elephant must be tall and slender, while the fellow who touched the beast's ear concluded that an elephant must be oblong and flat. Others of course reached different conclusions. The total result was that no man arrived at a very accurate description of the elephant. Yet each man had gained enough evidence from his own experience to disbelieve his fellows and to maintain a lively debate about the nature of the beast."
} 
Há duas categorias bem definidas de teorias sobre a UE: uma, referente ao processo de integração, ou seja, que busca explicar sua evolução, e outra, referente ao seu produto, o que busca explicar o que ela se tornou. ${ }^{19}$ Independentemente de seus desdobramentos, a distinção entre processo e produto permite perceber duas feições que nos interessam: uma dinâmica, referente à mútua transformação da comunidade e do Estado-membro, e outra estática, referente às estruturas comunitárias e nacionais que se instalam em razão da comunidade. Ambas se aplicam a todas as comunidades econômicas. Como veremos a seguir, observar a integração a partir de cada um desses pontos de vista revela algumas particularidades do tipo de arranjo que envolve as comunidades econômicas.

\subsection{Processo e produto por uma análise de spillover}

Vista como processo, a integração representa uma empreitada concreta, normalmente concebida em etapas de instalação, que transformam simultaneamente as estruturas nacionais e comunitárias para a construção da comunidade em si, ou seja, seu produto. Considerar o processo é, antes de tudo, focar no percurso, não tanto no seu destino.

Essa abordagem remete às teorias funcionalistas da integração, particularmente ao fenômeno do spillover. Essa maneira de conceber o processo de integração regional - inspirada no spillover de Haas (1958 apud MACCORMIK, OLSEN, 2014) e de Lindberg (1963 apud MACCORMIK, OLSEN, 2014) - considera-a um processo de unificação política, no qual dois ou mais Estados buscam o compartilhamento de algum tipo de estrutura jurídico-política. Por essa perspectiva, o nível de integração poderia ser colocado em uma escala teórica delimitada no extremo inferior pela ausência de integração (no qual os Estados ostentam autonomia recíproca absoluta) e no extremo superior pela integração total (do qual emerge um novo Estado, sucessor daqueles envolvidos no processo).

Essa escala imaginária pode ilustrar tanto o produto (um ponto na escala indicativo do grau ou profundidade de determinada comunidade) quanto o processo (um vetor na escala indicativo da direção, sentido e magnitude das transformações em curso). Segundo Haas e Lindberg (apud MACCORMICK, OLSEN, 2014), "[...] disparado o processo de integração, ele ganharia vida própria (uma 'lógica expansiva') pelo fenômeno de 'spillover '”, ${ }^{20}$ por meio do qual “[...] determinada

\footnotetext{
19 "Teorias que analisam a UE podem ser divididas em duas grandes categorias: aquelas que explicam como a UE evoluiu e aquelas que explicam o que ela se tornou. Enquanto a primeira categoria - que inclui as teorias de neofuncionalismo e intergovermentalismo - foi por muito tempo dominada por acadêmicos das relações internacionais, a segunda categoria vem crescentemente se tornando uma província dos acadêmicos de política comparativa. Esta última vê a UE como um sistema político com suas próprias instituições, processos, procedimentos e políticas. Em anos recentes o termo governança multinível tem emergido como uma maneira de entender a UE como um sistema político." Trad. livre de: "Theories analyzing the EU can be divided into two broad categories: those explaining how the EU evolved and those explaining what it has become. While the first categorywhich includes the theories of neofunctionalism and intergovernmentalism - was long dominated by scholars of international relations, the second category has increasingly become the province of scholars of comparative politics. The latter see the EU as a political system with its own institutions, processes, procedures, and policies. In recent years the term multilevel governance has emerged as one way to understand the EU as a political system." (MCCORMICK; OLSEN, 2014).

20 Trad. livre de: "He [HAAS] saw territorially based governing organizations as important 'agents of integration' and argued that once governments had launched the process of integration, it would take a life of its own (an 'expansive logic') through the phenomenon of 'spillover'."
} 
ação, relacionada a um objetivo específico, cria uma situação na qual o objetivo original só pode ser assegurado por meio de outras ações, que criam condições e necessidades para mais ações." (MACCORMICK, OLSEN, 2014). ${ }^{21}$ Extrapolando essa concepção, pode-se supor a existência de um motor intrínseco ao processo, ou seja, de um vetor que impulsiona a integração. McCormick e Olsen (2014) exemplificam:

Functional spillover implica que economias estão tão interconectadas que, se os estados integrarem um setor de suas economias, outros setores serão conduzidos à integração. OIGs especializadas teriam de ser criadas para supervisionar este processo, os poderes das instituições governamentais nacionais declinariam e haveria, eventualmente, união política e econômica.

Technical spillover implica que diferenças em standards levariam os estados a subir (ou baixar) para o nível daqueles com regulamentos mais rígidos (ou mais permissivos). Por exemplo, Grécia e Portugal - que tinham poucos controles ambientais antes de aderiram à UE - adotaram tais controles por conta dos requerimentos do direito da UE, que, por sua vez, era conduzido por pressões de estados com controles ambientais mais rígidos, como Alemanha e Holanda.

Political spillover implica que após a integração de diferentes setores operacionais, grupos de interesses (tais como lobby empresarial e sindicatos) deixaram de tentar influenciar os governos nacionais para tentar influenciar os governos regionais, que os encorajará a tentar conquistar novos poderes para eles próprios. Os grupos aproveitarão os benefícios da integração, e a política será cada vez mais disputada no nível regional em vez do nacional..$^{22}$

Com base nesse fenômeno, foram propostos pela literatura modelos de integração pelos quais haveria etapas a serem percorridas até o atingimento de uma união política total, seguindo mais ou menos a seguinte sequência: zona de comércio preferencial; zona de livre comércio; tarifa externa comum; união aduaneira; mercado comum; mercado único; união econômica com estabelecimento de moeda única e união política. ${ }^{23}$

Ainda que essas fases não representem um modelo canônico ou que o spillover não seja um fenômeno necessário, há em ambos lógica e pragmatismo suficientes para servir de base de raciocínio para a integração. Com essa abordagem, não é qualquer arranjo de base regional que se reputa integração, pelo menos não econômica, e não nesses termos. Arranjos políticos, ainda que discutam questões

\footnotetext{
21 Trad. livre de: Lindberg described this as a process by which 'a given action, related to a specific goal, creates a situation in which the original goal can be assured only by taking further actions, which in turn create a further conditions and need for further action' (LIND$B E R G$, 1963: p. 10)." Indicação de referência autor-data inexistente no corpo do texto original, senão em nota de fim.

22 Trad. livre de: "Functional spillover implies that economies are so integrated that if states integrate one sector of their economies, it will lead to the integration of other sectors. Functional IGOs would have to be created to oversee this process, the power of national government institutions would decline, and there would eventually be economic and political union [...] Technical spillover implies that disparities in standards will cause state to rise (or sink) to the level of those with the tightest (or loosest) regulations. For example, Greece and Portugal - which had few environmental controls because of the requirements of EU law, which in turn had been driven by pressures from states with tight environmental controls, such as Germany and the Netherlands. Political spillover implies that once different functional sectors are integrated, interested groups (such as corporate lobbies and labor unions) will switch from trying to influence regional institutions, which will encourage them in an attempt to win new powers for themselves. The groups will appreciate the benefits of integration, and politics will increasingly be played out at the regional rather than the national level."

23 A quantidade e estágio das etapas varia de autor para autor. Viljoen (2007, p. 485), por exemplo, descreve a fase de mercado comum (a qual considera o terceiro passo da integração) como a livre circulação de "bens, serviços e fatores de produção (capital e mão de obra)", enquanto alguns autores atribuem isso ao mercado único, distinguindo as duas fases, com base na evolução da União Europeia: a expressão "mercado único" refere-se ao Ato Único Europeu de 1986; a expressão "mercado comum" refere-se à Comunidade Econômica Europeia. Comparativamente, McCormick e Olsen (2014) identificam as seguintes fases: zona de livre comércio, união aduaneira, mercado comum, união econômica e união política.
} 
econômicas e que promovam coordenação constitucional entre os membros para atingir determinados objetivos, não se reputam como tais se não relacionados à consecução de algumas dessas fases.

Há, ainda, zonas cinzentas nesse assunto. Acordos que estabeleçam, por exemplo, tarifas reduzidas desafiam o conceito de integração. Dessa forma, até que ponto um tratado de tarifas reduzidas se reputa uma zona de comércio preferencial ou um tratado de isenção se reputa uma zona de livre comércio? Em acordos bilaterais tarifários, dúvidas a esse respeito são particularmente pertinentes, principalmente se considerado o caso dos Estados Unidos, que possui dezenas de acordos do tipo (UNITED STATES OF AMERICA, [20--]).

Certamente, mesmo acordos bilaterais tarifários colocam os mercados envolvidos em maior contato, havendo algum grau de integração. Mas esse tipo de arranjo não vai além do disposto nos tratados, não "disparando" o fenômeno de spillover. Além disso, em muitos casos, não há base regional, ainda que o conceito de região não se limite à contiguidade territorial. Por fim, tais acordos não envolvem organizações internacionais governamentais formalmente constituídas para lidar com as disposições do tratado. Não que isso descaracterize uma integração, se não como indício de eventualidade da parceria, que pode ser desfeita ao menor sinal de descontentamento por uma das partes.

Naturalmente, pode haver questões de outras ordens para a manutenção de acordos tarifários, impedindo, por motivos estratégicos, o desfazimento do vínculo. Mas essa situação não muda a extensão do acordo. Trata-se de discussão não da perspectiva do processo, mas do produto que se passa a analisar.

Sob a perspectiva de produto, a análise da integração concentra-se na sua imagem, ou melhor, na sua forma, remetendo a um dos estágios do seu processo. Outros aspectos também podem ser considerados, como tipo organizacional, estrutura institucional, etc. Recapitulando o conto de Puchala sobre o elefante, tentativas de enquadrar a comunidade em uma das categorias tradicionais pode levar a um reducionismo da sua "essência", fornecendo uma imagem distorcida da realidade, o que vai depender de cada caso.

Uma maneira abrangente de se descrever o tipo de empreitada diz respeito à distinção de shallow integration/deep integration: "[...] o conceito de deep integration é amplamente usado para se referir a qualquer arranjo que vá além de uma simples zona de livre comércio." (WORLD TRADE ORGANIZATION, 2011, p. 9). ${ }^{24}$ Por conseguinte, uma "simples zona de livre comércio" é o limite superior de um arranjo de shallow integration.

Definido nesses termos, os conceitos podem enganar, uma vez que não é a escala de integração que os define. Por exemplo, "[...] acordos preferenciais que incluam regras sobre políticas domésticas são considerados como 'deep agreements'." (WORLD TRADE ORGANIZATION, 2011, p. 9). ${ }^{25}$ Isto é, os conceitos de shallow e deep integration dizem respeito às matérias, limitando-se à fase de zona de livre comércio, já que na etapa seguinte (em que se estabelecem as tarifas externas comuns) logo são também consideradas políticas nacionais. Portanto, no caso dos tratados meramente tarifá-

\footnotetext{
${ }_{24}$ Trad. livre de: "[...] the concept of deep integration is widely used to refer to any arrangement that goes beyond a simple free trade area."

${ }_{25}$ Trad. livre de: "[...] preferential agreements that include rules on other domestic policies are referred to as 'deep' agreements."
} 
rios, bilaterais ou multilaterais, há apenas shallow integration. Arranjos da espécie não interessam ao presente estudo, já que, por definição, não há agenda de direitos humanos associada ao acordo, pois a mera existência de algo do tipo já denotaria um acordo de deep integration.

O conceito de deep integration pode ser utilizado para formular uma definição estrita de integração, pois acordos dessa natureza introduzem modificações nas políticas nacionais envolvidas para harmonização de setores determinados. Como corolário, a existência de OIGs deixa de ser determinante. Não obstante isto, não se analisam acordos de deep integration operacionalizados por meio de transconstitucionalismo ${ }^{26}$ (que não são formalizados internacionalmente), ${ }^{27}$ já que o objeto do presente estudo é justamente a organização estabelecida pela empreitada econômica.

Seguindo essa linha de raciocínio, a Comunidade Econômica Africana (AEC) pode finalmente ser enquadrada como organização política internacional governamental. Ora, o que há de concreto na atual situação da AEC são trabalhos de coordenação de outros projetos de integração, ou seja, de natureza transconstitucional relativamente às CERs eleitas como pilares da integração continental. À AEC não se reputa uma formalização internacional dessas comunidades, já que não há estruturas que as subordinem à AEC, não compondo entre elas sequer um sistema jurídico multinível.

\footnotetext{
${ }_{26}$ Transconstitucionalismo, conforme Neves (2014, p. 207): "Em face dessa situação, introduzo o conceito de transconstitucionalismo. Por um lado, o transconstitucionalismo não se confunde com um mero transjuridicismo, que pode ser observado inclusive na relação entre ordens jurídicas no pluralismo medieval, sobretudo entre direito canônico (e romano), direito urbano, direito real e direito feudal, pois, na experiência medieval, não se tratava de problemas constitucionais no sentido moderno, ou seja, nem de questões de direitos fundamentais nem de limitação e controle jurídico-positivo do poder, muito menos de pretensões diversas de autofundamentação do direito (em última instância e em geral, o direito tinha um fundamento sacro). Por outro lado, não se trata de constitucionalismo internacional, transnacional, supranacional, estatal ou local. O conceito aponta exatamente para o desenvolvimento de problemas jurídicos que perpassam os diversos tipos de ordens jurídicas. Um problema transconstitucional implica uma questão que poderá envolver tribunais estatais, internacionais, supranacionais e transnacionais (arbitrais), assim como instituições jurídicas locais nativas, na busca de sua solução. Para tratar do transconstitucionalismo, recorro ao conceito de 'razão transversal' de Wolfgang Welsch (1996; 2002, pp. 295-318), mas me afasto um tanto desse ambicioso conceito, para analisar os limites e possibilidades da existência de 'racionalidades transversais' ('pontes de transição') tanto entre o sistema jurídico e outros sistemas sociais (constituições transversais) quanto entre ordens jurídicas no interior do direito como sistema funcional da sociedade mundial."

27 Um fenômeno de regionalismo transconstitucional é aquele que se contrapõe a fenômenos de regionalismo formalizados por meio de instrumentos internacionais, dos simples tratados aos complexos acordos que envolvem a criação de organizações internacionais. Embora o surgimento da disciplina do regionalistmo esteja relacionado à proliferação de organizações intergovernamentais no pós-guerra, o fenômeno não se limita à forma, ao contrário: "Two points should be stressed. First, that regionalization is not based on the conscious policy of states or groups of states, nor does it presuppose any particular impact on the relations between the states of the region. And second, that patterns of regionalization do not necessarily coincide with the borders of states. Migration, markets, and social networks may lead to the increase interaction and interconnectedness tying together parts of existing states and creating new cross-border regions. The core of such 'transnational regionalism' may be economic as in the development of transborder growth triangles, industrial corridors, or the increasingly dense networks linking major industrial centres. Or it can be built around human interpentration, for example the transnational economic role played by overseas Chinese in East Asia or the dense societal linkages that now exist between California and Mexico." (HURRELL, 2003, p. 40). Considerando a definição de transconstitucionalismo de Neves na nota anterior, o 'regionalismo transnacional' de Hurrell (2003) cita exemplos de ragionalidades transversais entre sistema jurídico e outros sistemas sociais. Assim, haveria ainda uma segunda subespécie de regionalismo transconstitucional, baseado em racionalidades transversais entre "[...] ordens jurídicas no interior do direito funcional da sociedade mundial", isto é, que compreende "pontes de transição" estabelecidas por diálogos não formais entre ordens nacionais regionalmente vinculadas, a exemplo da região Ásia-Pacífico durante a guerra fria, que evitou a formalização e institucionalização do regionalismo para dificultar a influência americana na sua agenda econômica: "In Asia Pacific, by contrast, the far stronger states of the region have successfully resisted US efforts to promote APEC as an alternative formal vehicle for pressing its foreign econômica agenda. From their perspectiva a loose regional arrangement is a way of keeping the USA involved in the security of the region, whilst at the same time restricting its ability to press its economica agenda." (HURRELL, 2003, p. 51).
} 
A abordagem da integração como produto comporta, ainda, outras distinções, comparações e analogias, entre as quais a distinção (ou melhor, indistinção) estado/organização, que remete diretamente à escala imaginária proposta. A referência, mais uma vez, é a UE:

Por um lado, a UE possui qualidades de uma organização internacional: seus membros são estados nacionais, a adesão é voluntária, o balanço da soberania pende para os Estados-membros, a tomada de decisão é consultiva e os procedimentos baseiam-se no consenso ao invés da obrigatoriedade. Por outro, a UE também possui qualidade de estado: ela tem fronteiras internacionalmente reconhecidas (mesmo que estas tenham alterado de maneira realmente dramática em curto período de tempo em razão dos ciclos de crescimento da UE), há um sistema jurídico europeu ao qual todos os estados-membros se submetem, possui autoridade para influenciar e controlar a vida dos europeus, em muitas áreas o balanço de responsabilidade e poder deslocou para o nível europeu, e em algumas áreas - como o comércio - ela se tornou quase soberana e é reconhecida pelos outros estados como um ator equivalente. Contudo, ela não é nem uma nem outra, e tudo que podemos dizer com certeza - conforme já observado - é que ela é mais que uma organização internacional convencional e menos que um superestado europeu. (MCCORMICK; OLSEN, 2014). ${ }^{28}$

Essa observação revela a natureza híbrida da esfera comunitária, que orbita entre o nacional e o internacional, e justifica um conceito estrito de integração relacionado à deep integration. Parte-se da ideia que a integração emerge justamente dessa aproximação entre as esferas internacional e nacional, particularmente na transformação da OIG em um novo Estado quando da integração política total. Essa hibridez, por sua vez, é indício de diferenças substanciais entre CER e OPIG, capazes de interferir na condução de agendas de direitos humanos.

Ressaltar essa dupla feição da UE é necessário para quebrar a prevalência (na percepção jurídica) da qualidade de organização da UE e das demais CERs, cuja discussão, não raramente, tende a focar na distinção intergovernamental/supranacional, a qual nem representa categorias estanques - já que ambas podem coexistir em dada organização -, nem pacífica - já que não há um consenso sobre seu significado, particularmente em relação à UE. ${ }^{29}$ Críticas a essa distinção argumentam que ela "[...] desvia a atenção de um olhar sobre a UE como um sistema político a sua própria maneira." (MCCORMICK; OLSEN, 2014)..$^{30}$ Alternativamente, pode-se abordar a UE a partir da política comparativa ou de uma governança multinível:

Em vez de se deixar influenciar pela ótica das relações internacionais [...], talvez devamos olhar mais pelos métodos da política comparativa. Em 1994, Simon Hix argumentou que tais métodos poderiam nos ajudar a compreender como o poder governamental foi exercido na UE, como os europeus se relacionam com as instituições da UE e como o governo europeu foi influenciado por partidos políticos,

\footnotetext{
${ }_{28}$ Trad. livre de: "On the one hand, the EU also has some qualities of a state: it has internationally recognized boundaries (even if these have changed quite dramatically over a short period of time as the EU has gone through cycles of enlargement), there is a European system of law to which all member states are subject, it has increasing authority to influence and control the lives of Europeans, in many policy areas the balance of responsibility and power has shifted to the European level, and in some areas - such as trade -it has become all but sovereign and is recognized by other states as an equal player. However, it is neither one or the other, and all we can say with certainty - as noted earlier - is that it is more than a conventional international organization but less than a European superstate."

29 Cf. Mccormick e Olsen (2014).

30 Trad. livre de: "For its critics, the key problem with the intergovernmental/supranational date is that it treats the EU as an international organization and diverts attention away from looking at the EU as a political system in its own right."
} 
eleições e grupos de interesse. [...] Uma das limitações enfrentadas pela abordagem da política comparativa é a ausência de um governo europeu com contornos bem definidos. O termo governo aplica-se normalmente a instituições e agentes (eleitos ou apontados) que compõe a estrutura formal de governo de um estado e possui poderes para criar leis e definir a agenda política. Mas enquanto a UE possui um grupo de instituições de "governo" e muitos milhares de agentes contratados, não há um governo da UE como tal. Alternativamente, muitos preferem o uso do termo governança para descrever o sistema de autoridade da UE. Isso se refere a arranjos nos quais leis e políticas são elaboradas e implementadas sem a existência de um conjunto de instituições de governo formalmente reconhecidas, senão como resultado de interações envolvendo uma variável complexa de atores, incluindo o governo dos estados-membros, instituições da UE, grupos de interesse e outras fontes de influência. Levando essa ideia adiante, o termo governança multinível é usado para explicar como a UE está estruturada. Isso descreve um sistema no qual poder é compartilhado entre os níveis supranacional, nacional, subnacional e local, com considerável interação entre eles. (MCCORMICK; OLSEN, 2014, grifo do autor). ${ }^{31}$

Ambas as abordagens têm suas limitações, novamente em razão das peculiaridades que uma comunidade econômica adquire conforme se aproxima da forma estatal. Por um lado, a política comparativa enfrenta a dificuldade de lidar com o governo sui generis da esfera comunitária. Por outro, a governança multinível não é se não "[...] uma expressão mais sutil e complexa da ideia de federalismo" (MCCORMICK; OLSEN, 2014), ${ }^{32}$ descrevendo mais uma qualidade nacional. Não é uma surpresa, então, que entre as qualidades observáveis na UE, McCormick e Olsen (2014) também indiquem a federação e a confederação. De toda forma, a observação da indistinção possível entre Estado e OIG leva ao elemento final de diferenciação das CERs em relação aos sistemas de direitos humanos: enquanto nestas as OIGs poderiam ser vistas como fórum político interestatal, nas outras, a cada nível de aumento da integração, há maior semelhança da OIG com um Estado ou elemento unificador.

\section{Considerações finais: um balanço das diferenças}

Neste artigo partiu-se da preocupação de se compreenderem as diferenças tipológicas entre os Sistemas Internacionais de Direitos Humanos e as Comunidades Econômicas ao se direcionarem para a proteção de direitos humanos, atuação hoje em dia comum aos dois tipos de arranjos.

\footnotetext{
31 Trad. Livre de: "Instead of being so much influenced by the thinking of $I R$, they argue, perhaps we should be looking more at methods of comparative politics. In 1994, Simon Hix argued that those methods could help us understand how governmental power was exercised in the EU, how Europeans related to EU institutions, and how European government was influenced by political parties, elections, and interest groups. [...] One of the handicaps faced by comparative politics approaches is the absence of a clearly identifiable European government. The term government typically applies to the institutions and officials (elected or appointed) that make up the formal governing structure of a state and have the powers to make laws and set the formal political agenda. But while the EU has a group of 'governing' institutions and several thousand formally employed officials, there is no EU government as such. Instead, many prefer to use the term governance to describe the system of authority in the EU. This refers to an arrangement in which laws and policies are made and implemented without the existence of a formally acknowledged set of governing institutions, but instead as a result of interactions involving a complex variety of actors, including member state governments, EU institutions, interest groups, and other sources of influence. Taking this idea a step further, the term multilevel governance is used by some to explain how the EU is structures. This describes a system in which power is shared among the supranational, national, subnational, and local levels, with considerable interaction among them." 32 Trad. livre de: "In many ways, multilevel governance is just a more subtle and complex expression of the idea of federalism, which has been given relatively little attention by scholars of the EU."
} 
Uma vez esclarecida a diferenciação tipológica de cada um dos arranjos, torna-se possível, em uma nova agenda de pesquisa, uma análise mais aprofundada da relação entre integração regional e direitos humanos. Pode-se indagar como o processo de implantação da comunidade e o respectivo resultado afetam positiva e negativamente os direitos humanos, com o objetivo de identificar possíveis fatores de interferência da agenda econômica em uma agenda de direitos humanos. Ao ter em mente as diferenças tipológicas entre sistemas regionais de direitos humanos e comunidades econômicas regionais, concebe-se o ponto de partida para uma análise minuciosa das formas de diálogo entre as comunidades econômicas e os mecanismos dos sistemas de proteção, tanto no que se refere ao controle das atividades relacionadas à integração quanto à sobreposição de mecanismos e competências entre as duas esferas.

O ponto de partida foi responder a uma pergunta básica: o que constitui um 'sistema de direitos humanos'? Será que comunidades econômicas poderiam ser entendidas como tipos de sistemas de direitos humanos? O primeiro passo que levou à distinção das duas categorias foi a análise de três possíveis conceitos de sistema: como o objetivo de uma organização, como o maquinário jurídico-administrativo ou como um organograma interinstitucional. Concluiu-se que, enquanto a terceira acepção é uma mera coincidência de denominação, que não se liga necessariamente a direitos humanos, as duas primeiras estão inter-relacionadas. Assim, se um tratado fundador, que estabelece seu objetivo, é critério implícito de um sistema de direitos humanos, a noção de sistema de direitos humanos se confunde com a própria ideia de sua organização.

O segundo passo foi, então, investigar a natureza das organizações internacionais governamentais que correspondem a sistemas de direitos humanos, em busca de traços caracterizadores. Por um lado, um elemento distintivo é que os sistemas de direitos humanos são organizações políticas, enquanto a atividade das comunidades econômicas é de integração econômica. Por outro, a área de atuação das organizações internacionais correspondentes aos sistemas de direitos humanos pode variar, mas a presença de algum tipo de diálogo sempre está presente - o de conciliação, o de deliberação ou o de fomento. Esses dois elementos levam organizações internacionais governamentais - correspondentes a sistemas de direitos humanos - a se constituírem como um grande fórum político.

O terceiro passo foi explorar as grandezas dimensionais dos sistemas de direitos humanos, com especial comparação às comunidades econômicas regionais. Primeiro, os sistemas de direitos humanos tendem a apresentar maior correspondência com delimitações regionais, enquanto as comunidades econômicas têm escala sub-regional e são geralmente menores que os sistemas de direitos humanos em número de membros. Outro elemento de destaque é a relação entre as características dimensionais e a agenda de cada um dos arranjos. Enquanto uma organização política internacional governamental poderia comportar um número infinito de membros, o que não é limitado por seu objeto, as comunidades econômicas regionais possuem uma limitação imposta pelo próprio objeto por razões como o risco de expor um mercado comunitário.

Retomando as comunidades econômicas regionais como centro da análise, passou-se primeiro pelo caso paradigmático, que definiu historicamente a noção do que é uma comunidade 
econômica regional: a União Europeia. O caminho escolhido foi o de entender sua dinâmica de integração (seu processo), de um lado, e o que ela se tornou (seu produto), do outro. Por meio de uma análise de escala de spillover, identificou-se o que move o arranjo de integração econômica. De forma complementar, pensando em termos de shallow integration e deep integration, foi possível identificar que nas comunidades econômicas regionais, em que há o segundo tipo de integração, as organizações internacionais em si deixam de ser importantes quanto mais avançada a integração, já que se desloca cada vez mais para alterações de políticas nacionais. Da mesma forma, enquanto os sistemas de direitos humanos atuam como fórum político governamental, as comunidades econômicas regionais tendem a desenvolver uma unidade, o que diminui a importância da organização como locus de diálogo, transformando-a numa superestrutura semelhante a um Estado ou Confederação.

As diferenças pontuadas são fundamentais para auxiliar na reflexão futura sobre o novo locus de decisão sobre direitos humanos criado pela atuação - paralela à dos sistemas de direitos humanos - das comunidades econômicas regionais. Justamente por apresentarem agendas distintas (uma agenda política no caso dos sistemas de direitos humanos, e econômica no caso das comunidades econômicas), uma combinação dos achados dessa análise com uma observação mais atenta do que vem sendo a prática de comunidades econômicas regionais em direitos humanos - em comparação com a prática mais conhecida dos sistemas - permitirá verificar se há diferenças entre as racionalidades desses dois tipos de atores que influenciam em sua proteção dos direitos humanos.

\section{Referências}

ASSOCIAÇÃO DE NAÇÕES DO SUDESTE ASIÁTICO. Charter of the Association of Southeast Asian Nations. Singapura, 2007. Disponível em: < http://agreement.asean.org/media/download/20141204151618.pdf>. Acesso em: 23 jul. 2015.

COMUNIDADES ECONÔMICAS DOS ESTADOS DA ÁFRICA OCIDENTAL. ECOWAS Comission. Revised Treaty. Abuja: ECOWAS, 2010. Disponível em: <http://www.ecowas.int/wp-content/uploads/2015/01/Revised-treaty.pdf>. Acesso em: 24 ago. 2015.

COUNCIL OF EUROPE. Convention for the Protection of Human Rights and Fundamental Freedoms. European Treaty Series, Strasbourg, n. 5, 1950. Disponível em: < http://www.coe.int/en/web/ conventions/full-list//conventions/rms/0900001680063765>. Acesso em: 18 jan. 2016.

COUNCIL OF EUROPE. The Council of Europe: who we are? Strasbourg: Council of Europe, 2013. Disponível em: <https://edoc.coe.int/en/an-overview/5343-the-council-of-europe-who-we-are. html>. Acesso em: 23 jul. 2015.

EUROPEAN COMMUNITIES. Court of Justice of the European Communities. Case n. 1/58. Friedrich Stork \& Cie v. High Authority of the European Coal and Steel Community. Relator: Judge Riese. Luxemburgo, 04 Jan. 1959. Disponível em: <http://eur-lex.europa.eu/legal-content/EN/NOT/?uri=CELEX:61958CJ0001 > . Acesso em: 18 jan. 2016. 
EUROPEAN ECONOMIC COMMUNITY (EEC). Treaty Establishing The European Economic Community, 25 March 1957. Treaty Establishing The European Economic Community And Connected Documents. Luxembourg: Publishing Services Of The European Communities, [s.d.]. Disponível em: <http://www.cvce.eu/en/obj/treaty_establishing_the_european_economic_community_rome_25_march_1957-en-cca6ba28-0bf3-4ce6-8a76-6b0b3252696e.html>. Acesso em: 18 jan. 2015.

FAWCETT, Louise. Regionalism in Historical Perspective. In: FAWCETT, Louise; HURRELL, Andrew (Ed.). Regionalism in World Politics: Regional Organization and International Order. 1.ed. reimp. Oxford: Oxford University, 2003. p. 9-36.

FEFERBAUM, Marina. Sistemas sub-regionais de proteção dos direitos humanos: características e perspectivas. In: VEÇOSO, Fabia Fernandes Carvalho (Coord.). Direito Internacional em contexto. São Paulo: Saraiva, 2012. p. 213-282.

FREEMAN, Michael. Human Rights: An Interdisciplinary Approach. Cambridge: Cambridge University Press, 2002.

HURRELL, Andrew. Regionalism in Theoretical Perspective. In: FAWCETT, Louise; HURRELL, Andrew (Ed.). Regionalism in World Politics: Regional Organization and International Order. 1. ed. reimp. Oxford: Oxford University, 2003. p. 37-73.

LAURSEN, Finn. Theory and Practice of Regional Integration. Jean Monnet/Robert Schuman Paper Series, Miami, v. 8, i. 3, p. 3-22, Feb. 2008. Disponível em: <http://aei.pitt.edu/8219/1/LaursenLongSympos08RegIntegedi.pdf>. Acesso em: 18 jan. 2016.

LEAGUE OF ARAB STATES. Arab Charter On Human Rights, 22 May 2004. International Human Rights Reports, Nottingham, i. 12, 2005. Disponível em: <http://www1.umn.edu/humanrts/instree/leagueofarabstates.html>. Acesso em: 30 jul. 2015.

MCCORMICK, John; OLSEN, Jonathan. The European Union: politics and policies. 5. ed. Boulder: Westview, 2014. Versão e-book.

MERCOSUL. Conselho do Mercado Comum. Criação da reunião de altas autoridades sobre direitos humanos do Mercosul. Decisão n. 40/04, de 16 dezembro 2004. Disponível em: < http://gd. mercosur.int/SAM/GestDoc/pubweb.nsf/Normativa?ReadForm\&lang=POR\&id=09C40D3856EB84E00325760F006FDF77>. Acesso em: 16 ago. 2015.

MERCOSUL. Conselho do Mercado Comum. Instituto de Políticas Públicas de Direitos Humanos. Decisão n. 14/09, de 23 de julho de 2009. Disponível em: < http://gd.mercosur.int/SAM/GestDoc/ pubweb.nsf/Normativa?ReadForm\&lang=POR\&id=C1EB549352CDD5620325760000679C9F $>$. Acesso em: 16 ago. 2015.

MURUNGI, Lucyline Nkatha; GALLINETTI, Jacqui. The Role of Sub-Regional Courts in the African Human Rights System. Sur, International Journal on Human Rights - English Edition, São Paulo, v. 7, i. 13, p. 119-143, Dec. 2010. Disponível em: <http://www.conectas.org/Arquivos/edicao/publicacoes/publicacao-201424174111209-53513886.pdf>. Acesso em: 18 jan. 2016.

NEVES, Marcelo. (Não) solucionando problemas constitucionais: transconstitucionalismo além de colisões. Lua Nova, São Paulo, v. 93, p. 201-232, 2014. 
O’DONNELL, Guillermo. Delegative Democracy? Kellogg Institute Working Paper, Notre Dame, i. 192, p. 1-17, Apr. 1993. Disponível em: <https://kellogg.nd.edu/publications/workingpapers/ WPS/172.pdf>. Acesso em: 04 ago. 2015.

ORGANIZATION OF AFRICAN UNITY (OAU). African Charter On Human And Peoples' Rights, 27 June 1981. OAU Doc. Cab/leg/67/3 Rev. 5., reimpresso em International Legal Materials, Washington, v. 21, i. 21, 1982. Disponível em: <http://www.achpr.org/instruments/achpr/>. Acesso em: 18 jan. 2016.

ORGANIZATION OF AMERICAN STATES (OAS). American Convention on Human Rights "Pact of San Jose, Costa Rica", 22 November 1969. Washington, 1969. Disponível em: <http://www.oas.org/ dil/treaties_B-32_American_Convention_on_Human_Rights.pdf >. Acesso em: 18 jan. 2016.

PIOVESAN, Flávia. Direitos Humanos e o Direito Constitucional Internacional. 10. ed., rev. e atual. São Paulo: Saraiva, 2009.

PUCHALA, Donald J. Of Blind Men, Elephants and International Integration. JCMS: Journal of Common Market Studies, v. 10, i. 3, p. 267-284, mar. 1972. doi: 10.1111/j.1468-5965.1972. tb00903.x. Disponível em: <http://onlinelibrary.wiley.com/doi/10.1111/j.1468-5965.1972. tb00903.x/abstract>. Acesso em: 18 jan. 2016.

SEN, Amartya. Desenvolvimento como liberdade. São Paulo: Companhia das Letras, 2000.

SOUTHERN AFRICAN DEVELOPMENT COMMUNITY. Treaty of the Southern African Development Community. 17 ago. 1992. Disponível em: <http://www.sadc.int/documents-publications/ show/865>. Acesso em: 24 ago. 2015.

UNIÃO EUROPEIA. Tratado Euratom Versão Consolidada. Luxemburgo: Serviços das Publicações da União Europeia, 2010. Disponível em: <http://europa.eu/eu-law/decision-making/treaties/pdf/ consolidated_version_of_the_treaty_establishing_the_european_atomic_energy_community/consolidated_version_of_the_treaty_establishing_the_european_atomic_energy_community_pt.pdf $>$. Acesso em: 18 jan. $20 \overline{16}$.

UNITED NATIONS. 2013 Demographic Yearbook. 64. ed. New York: United Nations, 2014. Disponível em: <http://unstats.un.org/unsd/demographic/products/dyb/dybsets/2013.pdf>. Acesso em: 23 jul. 2015.

UNITED NATIONS. Funds, Programmes, Specialized Agencies and Others. 2015. Disponível em: <http://www.un.org/en/aboutun/copyright/index.html>. Acesso em: 28 jul. 2015.

UNITED NATIONS. Resolution n. 2200 A (XXI), 16 December 1966. International Covenant on Civil and Political Rights. Resolutions Adopted By The General Assembly During Its 21st Session. New York, 1967a, Seção A/6316, p. 52-58. Disponível em: <http://daccess-dds-ny.un.org/doc/RESOLUTION/GEN/NR0/005/03/IMG/NR000503.pdf?OpenElement>. Acesso em: 18 jan. 2016.

UNITED NATIONS. Resolution n. 2200 A (XXI), 16 December 1966. International Covenant on Economic, Social and Cultural Rights. Resolutions Adopted By The General Assembly During Its 21st Session. New York, 1967b, Seção A/6316, p. 49-52. Disponível em: <http://daccess-dds-ny. un.org/doc/RESOLUTION/GEN/NR0/005/03/IMG/NR000503.pdf?OpenElement>. Acesso em: 18 jan. 2016. 
UNITED NATIONS. Resolution n. 34, 18 December 1979. Convention on the Elimination of All Forms of Discrimination Against Women. Resolutions And Decisions Adopted By The General Assembly During Its 34th Session. New York, 1980, Seção A/34/46. p. 193-198. Disponível em: <http://daccess-dds-ny.un.org/doc/RESOLUTION/GEN/NR0/378/07/IMG/NR037807.pdf?OpenElement>. Acesso em: 18 jan. 2016.

UNITED STATES OF AMERICA. U.S. Department Of State. Office Of The Historian. Wilson's Fourteen Points, 1918. [20--]. Disponível em: <https://history.state.gov/milestones/1914-1920/ fourteen-points>. Acesso em: 10 jul. 2015.

VILJOEN, Frans. International Humam Rights Law in Africa. Oxford: Oxford University, 2007.

VILJOEN, Frans. International Humam Rights Law in Africa. 2. ed. Oxford: Oxford University, 2012.

WORLD TRADE ORGANIZATION. World trade report 2011.

Data de submissão: 04 de maio de 2016 Avaliado em: 07 de setembro de 2016 (AVALIADOR A) Avaliado em: 02 de outubro de 2016 (AVALIADOR B) Aceito em: 04 de novembro de 2016 
